\title{
SINTOMATOLOGIA OCUPACIONAL E QUALIDADE DE VIDA EM COSTUREIROS (AS) DE UMA FÁBRICA DE CONFECÇÕES
}

\author{
Maria Juliana Ferreira dos Santos ${ }^{1}$ \\ Pâmala Samara Formiga ${ }^{2}$ \\ Emanuely Rolim Nogueira ${ }^{3}$ \\ Juliane Carla Medeiros de Sousa ${ }^{4}$ \\ Elisangela Vilar de Assis ${ }^{5}$ \\ Michel Jorge Dias ${ }^{6}$
}

RESUMO: OBJETIVO: Verificar a prevalência da sintomatologia ocupacional e a qualidade de vida de costureiros (as) de uma fábrica de confecções. MÉTODO: Trata-se de um estudo transversal, descritivo com abordagem quantitativa, composto por 12 costureiros/as de uma fábrica de confecções na cidade de Tenente Ananias-RN, sendo a amostra por conveniência e aleatória, desenvolvida nos meses de setembro e outubro de 2018. Foi aplicado um questionário contendo dados sobre o perfil sociodemográficos e perguntas sobre questões de trabalho. Para avaliação das dores utilizou-se o Diagrama de Posturas de Corlett e Manenica. Para avaliação da qualidade de vida foi utilizado o questionário de Estado de Saúde SF-36v2. Os dados foram submetidos aos testes de Shapiro Wilk e Levene. RESULTADOS: O estudo revelou que todos os participantes auto relataram dores musculares em locais distintos do corpo. Observou-se predomínio da algia na região da coluna lombar $(41,7 \%)$ e nos pés $(41,66 \%)$. Com relação à qualidade de vida, o maior índice encontrado foi no domínio limitação por aspectos físicos com uma média de 93,25, seguido pelo domínio aspectos sociais $(63,50)$ e em terceiro ficou o estado geral de saúde $(56,8)$. CONCLUSÃO: Os participantes são acometidos por algias com diferentes intensidades em alguma parte do corpo, ocasionando desconfortos físicos e psicológicos. A qualidade de vida se caracterizou como boa, sendo

\footnotetext{
${ }^{1}$ Graduanda do curso Bacharelado em Fisioterapia pela Faculdade Santa Maria, Cajazeiras PB - Email: mariajuliana.mjf@gmail.com.

${ }^{2}$ Fisioterapeuta. Graduada pela Faculdade Santa Maria, Cajazeiras-PB, E-mail: pamala.samara@outlook.com.

${ }^{3}$ Fisioterapeuta. Especialista. Docente da Faculdade Santa Maria, Cajazeiras-PB, E-mail: emanuelyfisio@gmail.com.

${ }^{4}$ Fisioterapeuta. Mestre. Docente da Faculdade Santa Maria, Cajazeiras-PB, E-mail: julianecarlam@gmail.com.

${ }^{5}$ Fisioterapeuta. Doutora. Docente da Universidade Federal de Campina Grande, Cajazeiras-PB, Email: ely.vilar@hotmail.com.

${ }^{6}$ Fisioterapeuta. Mestre. Docente da Faculdade Santa Maria, Cajazeiras-PB, E-mail: michelj_dias@hotmail.com.
} 
evidenciadas queixas e sintomatologias que podem influenciar posteriormente na capacidade para o trabalho, assim, favorecendo o prejuízo na saúde, consequentemente na qualidade de vida.

PALAVRAS CHAVE: Doenças ocupacionais. Qualidade de vida. Saúde do trabalhador.

\section{INTRODUÇÃO}

As constantes transformações no ambiente de trabalho nas empresas possuem o objetivo de alcançar metas e alavancar suas produtividades, considerando a necessidade de produção e serviços de alta qualidade, desenvolvem um mercado cada vez mais competitivo levando os seus funcionários aos seus limites físicos e psicossociais. Na mesma proporção, o oferecimento de condições adequadas aos trabalhadores na tentativa de aumentar a produtividade $\mathrm{e}$ lucratividade e diminuir os riscos fornecidos e o aparecimento de doenças se torna muito necessário no mundo atual devido ao cenário capitalista que a maioria dos trabalhadores estão envolvidos (VERONESI JUNIOR, 2014).

Os problemas de saúde decorrentes da relação de trabalho têm se tornado um fenômeno mundial, destacando-se, assim, aqueles relacionados às doenças ocupacionais, denominados de lesões por esforços repetitivos (LER) e distúrbios osteomusculares relacionados ao trabalho (DORT). Esses distúrbios são caracterizados pela incapacidade laboral, temporária ou permanente, resultante da combinação de sobrecarga do sistema osteomuscular com falta de tempo para recuperação dos músculos, podendo causar limitação funcional e transtorno psicossocial (MORETTO, 2017).

Os principais sintomas característicos tanto das DORTs, quanto da LER são: dor, parestesia, sensação de peso, fadiga e antipatia (VERONESI JUNIOR, 2014). A dor, como principal sintoma é de difícil manejo pelos profissionais da saúde devido a sua complexidade; está associada não apenas a uma lesão orgânica e física, mas sim a outros fatores que propiciam seu agravo, como aspectos cognitivos, culturais e 
emocionais, influenciando no bem-estar e na saúde do trabalhador no seu ambiente de trabalho (APKARIAN; BALIKI; GEHA, 2009).

O trabalho de costureiras por utilizarem os mesmos grupamentos musculares de forma repetitiva e no ritmo acelerado em longas horas de trabalho e posturas incorretas estão sujeitas a uma maior incidência da dor, pincipalmente na região dorsal, lombar, costas, antebraços e mãos (AMBROSI; QUEIROZ, 2004). Assim, esse fator influencia na qualidade de vida desses profissionais bem como a sua saúde de forma direta ou indiretamente, desenvolvendo como consequência o absenteísmo e limitação de suas habilidades no ambiente de trabalho (INOUE; MATSUDA; SILVA, 2008).

A má circulação sanguínea e cansaços nos membros superiores e inferiores constituem também fatores que acabam influenciando na qualidade de vida desses profissionais, onde necessitam de um esforço muscular, boa visão, concentração mental, e por ser uma atividade monótona e repetitiva acaba não gerando bons resultados dentro do ambiente de trabalho, os quais obrigam muitas vezes a permanecer por muito tempo trabalhando, de uma forma pouco ajustada e com um rigoroso sistema de qualidade da produção, levando ao desenvolvimento de problemas e sofrimento ao trabalhador (ROSA; PILATTI, 2007).

Essas considerações são reforçadas por Prado (2006), quando afirma que as exigências do trabalho na indústria de confecção juntamente com a configuração da postura dos funcionários em seu posto de trabalho, o estresse em relação à exigência de produtividade, ruídos e temperaturas desagradáveis, podem favorecer a caracterização de uma profissão altamente fatigante.

Este estudo se justifica pelo fato do setor de costura da indústria de confecção apresentar um ambiente de trabalho que pode ocasionar desconfortos e danos à saúde do trabalhador, como também influenciar na sua qualidade de vida, devido às atividades laborais serem altamente repetitivas, monótonas e ainda serem realizadas na mesma posição durante toda a jornada de trabalho.

Tendo como principal objetivo verificar a prevalência da sintomatologia ocupacional e a qualidade de vida de costureiros (as) de uma fábrica de confecções. 


\section{MÉTODO}

Trata-se de uma pesquisa transversal, analítica com abordagem quantitativa, composta por 12 costureiras de uma fábrica de confecções na cidade de Tenente Ananias-RN. Foi desenvolvida nos meses de setembro e outubro de 2018 , sendo a amostra selecionada de forma aleatória e por conveniência.

Foram incluídos trabalhadores com idade a partir de 18 anos, de ambos os sexos, com ocupação exclusiva de costureiros/costureiras, há pelo menos um ano, e excluídos da pesquisa os trabalhadores de licença e de férias, que não trabalhavam de forma integral na empresa, que já tivessem doenças existentes auto relatadas antes de começarem a trabalhar na indústria de confecção.

Para coleta de dados foi utilizado um questionário elaborado pelos pesquisadores em que constou de dados sobre o perfil sociodemográfico (sexo, idade, escolaridade); perguntas sobre o trabalho (turno e jornada, horas de descanso).

Utilizou-se o questionário de Diagrama de Posturas de Corlett e Manenica (1980) validado na Universidade de Nottinghan - 1993 e semelhante ao método Rapid Upper Limb Assessment - RULA (SOUZA; MAZINI FILHO 2017). O Diagrama de Corlett e Manenica foi aplicado solicitando aos usuários do ambiente, neste caso as costureiras, que indicassem a região e a intensidade de desconforto que sentiam após terem realizado a tarefa na fábrica de costura. Esse diagrama é constituído por um questionário, que divide o corpo em regiões onde o sujeito pode sentir dor, e que admite cinco respostas para a intensidade de desconforto/dor em cada parte do corpo: (1) nenhum (2) algum desconforto, (3) moderado, (4) bastante e (5) extremo (LIGEIRO, 2010).

Para a análise da qualidade de vida foi utilizado o questionário de Estado de Saúde SF-36V2, traduzido e validado por Cicoleni et al. (1999). Para a pontuação dos itens, foi utilizada uma escala tipo Raw Escale de oito pontos para analisar o nível de concordância dos respondentes com os itens expostos no instrumento de coleta de dados. Os participantes que apresentaram índices abaixo de 20 foram 
consideradas pior estado de saúde; aqueles com níveis entre 20 a 40 regular, de 40 a 60 tiveram nível bom; aqueles com 60 a 80 nível muito bom e colaboradoras que atingiram 80 a 100 foram considerados níveis excelentes de qualidade de vida (SILVA, 2015).

A análise estatística dos dados foi realizada no Programa Microsoft Excel (2013), de forma descritiva, em que as variáveis quantitativas foram apresentadas por meio de médias e desvios-padrão. Com relação aos dados a respeito da qualidade de vida das costureiras, foi identificado que se encontram dentro da normalidade e da homogeneidade, e os mesmos foram submetidos aos testes de Shapiro Wilk e Levene, para analisar a normalidade dos dados e para verificar se os dados apresentavam homogeneidade de variância respectivamente. Foi adotado um intervalo de confiança de $95 \%$.

Os participantes assinaram o Termo de Consentimento Livre e Esclarecido TCLE, a coleta de dados ocorreu conforme aprovação do estudo pelo Comitê de Ética e Pesquisa (CEP) da Faculdade Santa Maria (FSM), com o número do parecer: 2.906.014, e seguiu as normas da resolução 466/12 do Conselho Nacional de Saúde - Pesquisa envolvendo seres humanos (BRASIL, 2012).

\section{RESULTADOS E DISCUSSÃO}

A amostra do estudo foi composta por 12 (doze) costureiros (as), com prevalência para o sexo feminino $(91,7 \%)$. Com relação à idade a predominância foi para as participantes que se enquadravam na categoria de 30 a 50 anos (75\%). Quanto o grau de escolaridade, $66,8 \%$ dos respondentes afirmou possuir $1^{\circ} \mathrm{grau}$ incompleto, e no que diz respeito a remuneração, todos os participantes relataram receber de 1 a 2 salários mínimos.

Dentre as variáveis observadas foi constatado que todos os entrevistados relataram trabalhar de forma rápida, sendo associado com a exigência do esforço físico com movimentos rápidos $(58,3 \%)$, posicionamento dos braços e das pernas de forma inadequada $(83,3 \%)$ e posição fisicamente inadequada, desconfortável, com 
66.7\%. Ainda sobre a exigência da posição física, 83,33\% dos entrevistados possuíam uma posição inadequada tanto dos braços quanto das pernas.

O dado evidenciado de esforços físicos rápidos corrobora com o estudo, sobre prevalência e fatores associados à sintomatologia dolorosa entre profissionais da indústria têxtil, no qual relata que durante a jornada de trabalho, a maioria dos indivíduos que executava movimentos repetitivos e rápidos, e não realizavam pausas para descanso do segmento do corpo (MACIEL; FERNANDES; MEDEIROS, 2006).

Ainda sobre a exigência da posição física, a grande maioria dos entrevistados possui uma posição inadequada tanto dos braços quanto das pernas, e essas alterações podem ser evidenciadas no estudo de Ferreira, Silva, Vasconcelos (2016) sobre desconfortos musculoesqueléticos em costureiras, onde relatam o predomínio desta posição inadequada dos membros, afirmando também que a atividade laboral associada aos esforços repetitivos das costureiras, o tempo prolongado na mesma postura, são alguns dos fatores que intervém negativamente na qualidade de vida e no estado emocional dos trabalhadores. E a presença destes fatores, isolados ou em conjunto, podem favorecer a ocorrência de dores musculares.

Foi constatado que todos os entrevistados trabalham pela manhã e tarde, e com jornada de trabalho maior do que oito horas diárias. Do tempo de trabalho, $50 \%$ trabalham a mais cinco anos, e 75\% relataram ter tempo para descanso.

Siqueira (2010) relata que devido à exaustão dos esforços físicos realizados no trabalho de confecções, muitos são os problemas encontrados nesses trabalhadores e que, devido a essa circunstância, acabam se afastando do serviço por causa de inúmeros problemas de saúde, principalmente relacionado a dores.

A pesquisa revelou que todos os participantes apresentavam dores musculares em locais distintos do corpo. De acordo com a presença de dor em tronco, membros superiores e membros inferiores, observou-se o predomínio de algia na região da coluna lombar, totalizando $41,7 \%$, ombros $33,33 \%$ e pés com $41,66 \%$.

O estudo evidenciou inúmeras áreas corporais com relato de dor frequente e/ou incômodas, sendo que a região lombar e pé foram às áreas mais afetadas. Este dado não condiz com o estudo de Zago, Piccoli, Renner (2012) no qual, as médias 
do nível de desconforto/do são baixas e se encontram todas na classificação de dor leve.

O gráfico 1 evidencia a intensidade das dores nas últimas 4 semanas, onde foi observando o predomínio de dores moderadas $50 \%$.

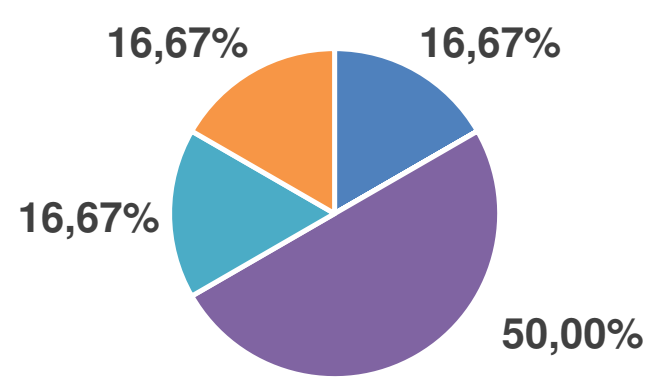

- Nenhuma $=$ Muito fracas $=$ Ligeiras $\|$ Moderadas $\|$ Fortes $=$ Muito fortes

Gráfico 1 - Intensidade da dor nas últimas 4 semanas.

Podemos observar que prevaleceu entre as respostas das costureiras as dores moderadas. Concordando, Souza (2015) relata que presença das dores são comuns e que a possibilidade dessas dores pode estar relacionada a comprometimento osteomuscular em algumas trabalhadoras.

A tabela 1 apresenta a descrição em média, desvio padrão, valor mínimo e valor máximo, intervalo de confiança e índices por domínio da qualidade de vida encontrados nas costureiras. 


\begin{tabular}{|c|c|c|c|c|c|c|}
\hline \multirow{2}{*}{ Domínios } & \multirow{2}{*}{ Média } & \multirow{2}{*}{$\begin{array}{l}\text { Desvio } \\
\text { Padrão }\end{array}$} & \multirow{2}{*}{$\begin{array}{l}\text { Valor } \\
\text { Mínimo }\end{array}$} & \multirow{2}{*}{$\begin{array}{l}\text { Valor } \\
\text { Máximo }\end{array}$} & \multicolumn{2}{|c|}{$\begin{array}{l}\text { Intervalos de } \\
\text { Confiança 95\% }\end{array}$} \\
\hline & & & & & Limite Inf. & $\begin{array}{l}\text { Limite } \\
\text { Sup. }\end{array}$ \\
\hline $\begin{array}{l}\text { Estado geral de } \\
\text { saúde }\end{array}$ & 56,80 & 0,83 & 16 & 4,2 & 56,6 & 56,9 \\
\hline $\begin{array}{l}\text { Capacidade } \\
\text { funcional }\end{array}$ & 55,4 & 0,84 & 15 & 3 & 55,26 & 55,54 \\
\hline $\begin{array}{l}\text { Limitação por } \\
\text { aspectos } \\
\text { físicos }\end{array}$ & 93,25 & 1,37 & 10 & 5 & 93,03 & 93,47 \\
\hline Dor & 42,22 & 1,09 & 10 & 6 & 42,04 & 42,4 \\
\hline Vitalidade & 34,55 & 0,98 & 1 & 4 & 34,79 & 34,71 \\
\hline $\begin{array}{l}\text { Aspectos } \\
\text { sociais }\end{array}$ & 63,50 & 0,66 & 2 & 5 & 63,68 & 63,62 \\
\hline $\begin{array}{l}\text { Limitação por } \\
\text { aspectos } \\
\text { sociais }\end{array}$ & 12,66 & 1,33 & 1 & 5 & 12,44 & 12,88 \\
\hline Saúde mental & 53,64 & 1,065 & 1 & 5 & 53,33 & 53,82 \\
\hline Qv. Total & 51,50 & 1,02 & 1 & 5 & 51,33 & 51,67 \\
\hline
\end{tabular}

Tabela 1 - Domínios da qualidade de vida.

Portanto, de acordo com a média de correlação dos domínios, a qualidade de vida dos trabalhadores se configura com um bom nível. De acordo com os dados observados, o maior índice encontrado foi no domínio "limitação por aspectos físicos" com uma média de 93,25, seguido pelo domínio "aspectos sociais" com 63,50 e em terceiro ficou o "estado geral de saúde" com 56,8.

No estudo de Alcântara et al. (2014) a respeito de doenças relacionadas ao trabalho, foi observado que a limitação física chega a impedir a execução de atividades rotineiras, de cuidados pessoais e domésticos. Assim, os trabalhadores passam a depender de outras pessoas pela incapacidade de alimentar-se e vestirse. O autor ainda reforça dizendo que se a atividade é monótona, repetitiva, composta de tarefas de execução, sem espaço para concepção ou decisão e com pressão sobre a produtividade, as consequências refletem sobre o corpo, gerando comprometimento físico e diminuição da produtividade que é tão cobrada. 
Estudos mostram que esses trabalhadores são submetidos frequentemente a níveis elevados de estresse devido à sobrecarga e pressão pela produtividade e cumprimento de prazos, causando transtornos mentais, como a depressão, que é um dos maiores motivos de afastamento do trabalho entre as mulheres (VALENÇA; FERRAZA; LOPES, 2016).

\section{CONCLUSÃO}

Este estudo mostra que esses trabalhadores (costureiros/as) de confecções sentem dor ou incômodos com diferentes intensidades em alguma parte do corpo, ocasionando desconfortos físicos e psicológicos. Apesar desses achados, a qualidade de vida desses indivíduos se caracterizou como boa.

$O$ estudo evidenciou queixas e sintomatologias que podem influenciar, com o passar do tempo, a capacidade para o trabalho dessas profissionais. É possível observar que as costureiras estão predispostas a vários fatores agressivos ao organismo, assim, favorecendo o prejuízo na sua saúde, consequentemente na sua qualidade de vida.

\section{REFERÊNCIAS BIBLIOGRÁFICAS}

ALCANTARA, F.L.D. et al. Busca por uma melhor qualidade de vida no trabalho utilizando a ginástica laboral como ferramenta. Seget, 2014.

AMBROSI, D.; QUEIROZ, M.F.F. Compreendendo o trabalho da costureira: um enfoque para a postura sentada. Rev Bras Saúde Ocup. v. 29, n.109, p.11-9, 2004.

APKARIAN, A.V., BALIKI, M.N., GEHA, P.Y. Towards a theory of chronic pain. Prog Neurobiol. v.87, n.2, p.81-97, 2009.

BRASIL. Ministério da Saúde. Resolução 466/12 do Conselho Nacional de Saúde, sobre as diretrizes e normas regulamentadoras de pesquisa envolvendo seres humanos. Diário oficial da união, 13 de junho de 2012.

FERREIRA, A.P.B.; SILVA, M.R.O.; VASCONCELOS, L.A.V. Desconforto musculoesquelético em costureiras: uma análise comparativa. Rev. Trab. Acad., v. 3, n.3, 2016.

INOUE, K.C.; MATSUDA, L.M.; SILVA, D.M.P.P. Absenteísmo em unidade de terapia intensiva de um hospital-escola. Revista Ciência, Cuidado e Saúde, Maringá, n.7, p.11-17. 2008. 
LIGEIRO, J. Ferramentas de avaliação ergonômica em atividades multifuncionais: a contribuição da Ergonomia para o design de ambientes de trabalho. [Dissertação apresentada ao Programa de Pós Graduação em Design da Universidade Estadual Paulista Julio de Mesquita Filho] Bauru, Unesp, 2010.

MACIEL, A.C.C.; FERNANDES, M.B.; MEDEIROS, L.S. Prevalência e fatores associados à sintomatologia dolorosa entre profissionais da indústria têxtil. Rev. bras. epidemiol., São Paulo, v. 9, n. 1, p. 94-102, 2006.

MORETTO, A.F.; CHENASI, F.H.; GRILLO, L.P. Sintomas osteomusculares e qualidade de vida em costureiras do município de Indaial, Santa Catarina. Fisioter Pesqui.; v. 24, n. 2, p. 163168, 2017.

PRADO, R.R. Avaliação da qualidade de vida na indústria do vestuário: o caso de costureiras portadoras de lombalgias. [Dissertação: mestrado em Desenho Industrial - Área de Concentração: Ergonomia] Universidade Estadual Paulista - Faculdade de Engenharia, f.98, Bauru, 2006.

ROSA, M.A.S.; PILATTI, L.A. Qualidade de vida no trabalho: análise do caso de colaboradores de uma empresa do ramo de metalúrgica de Ponta Grossa - PR. Lecturas, Educación Física y Deportes, Rev. digital. [periódico na internet], Maio 2007.

SILVA, F.G. Análise da dor e qualidade de vida (qv) em costureiras de confecções de pequeno porte na cidade de Ervália, MG. Anais VI SIMPAC, Viçosa-MG, v. 6, n. 1, p. 65-70, jan/dez. 2014.

SIQUEIRA, A.F. Avaliação ergônomica do posto de trabalho: apresentação de metodologias como suporte a propostas de melhorias. Simepro, 2010.

SOUZA, J.A.C.; MAZINI FILHO, M.L. Análise ergonômica dos movimentos e posturas dos operadores de checkout em um supermercado localizado na cidade de Cataguases, Minas Gerais. Gest. Prod., São Carlos, v. 24, n. 1, p. 123-135, Apr. 2017.

SOUZA, K.V.L.; NETO, M.G. Análise da Qualidade de Vida e Distúrbios Osteomusculares dos Funcionários Administrativos de um Órgão Público. Revista Pesquisa em Fisioterapia, Dez; v. 5, n. 3, 2015.

VALENÇA. J.B.M., FERRAZA, K.P., DE ALENCARA, M.C.B., SOUZA, F.G., LOPES, L.V. Perfil de trabalhadores com doenças da coluna vertebral atendidos em um serviço de saúde. Cad Ter Ocup. UFSCar, São Carlos. v. 24, n.2, p.227-33, 2016.

VERONESI JUNIOR, J.R. Fisioterapia do trabalho: cuidado da saúde funcional do trabalhador. São Paulo: Ed. Andreoli, $2^{\circ}$ Ed. p.47-48, 2014.

ZAGO, A.T, PICCOLI, J.C.J. RENNER, S.J. Prevalência de desconfortos na coluna vertebral e fatores associados em costureiras do setor calçadista. Rev, digit, Buenos aeres, v.5, p,40-41, 2012. 